\title{
Recurrent Pancreatic Acinar Cell Carcinoma
}

National Cancer Institute

\section{Source}

National Cancer Institute. Recurrent Pancreatic Acinar Cell Carcinoma. NCI Thesaurus. Code C153569.

The reemergence of pancreatic acinar cell carcinoma after a period of remission. 